\section{Importance of anti- and pro- nociceptive mechanisms in human disease}

\section{Tracey, P Dunckley}

\section{Abnormalities in endogenous descending pain inhibitory and facilitatory influences probably contribute to the development and maintenance of chronic pain states}

$\mathrm{T}^{\mathrm{T}}$ he burden of chronic pain to society is enormous. This is both in terms of physical and emotional impact to individuals and carers, in addition to the large financial burden. Current estimates suggest that $11.5-55.2 \%$ of individuals worldwide are defined as suffering from chronic widespread pain. ${ }^{1}$ A major characteristic of functional disorders such as irritable bowel syndrome (IBS) and inflammatory/ neuropathic disorders such as gastrooesophageal reflux and chronic pancreatitis is abdominal discomfort or pain. There is an increasing awareness that many similarities exist mechanistically between somatic chronic pain conditions and the pain witnessed as chronic in IBS and chronic pancreatitis patients. With this realisation there has been a change of focus for researchers of both somatic and visceral pain conditions from peripheral structures as the preferred target of research to the central nervous system (CNS). It has long been recognised that the CNS has a major modulating nociceptive influence that alters resultant pain perception. ${ }^{2-4}$ Recent developments in neuroimaging have enabled CNS investigations of visceral pain processing in patients and controls and such studies have highlighted the additional relevance of cognitive and emotional factors in modulating pain perception from physical changes such as plasticity and sensitisation. $^{5-7}$ Imaging studies have provided valuable objective information on what is inherently a subjective phenomenon, that for too long has relied upon patients giving a self report of their pain using coarse pain rating scales. ${ }^{8}$ Currently, there is a wider imaging literature on pain processing from somatic structures compared with visceral organs. This is probably because it is experimentally (and ethically) easier to perform somatic acute pain paradigms in healthy controls (for instance, using noxious thermal events) compared with more challenging oesophageal or rectal balloon distensions. However, this situation is rapidly changing and in terms of investigating relevant patient groups with pain conditions, there is a rapidly growing literature investigating visceral pain syndromes that competes with imaging studies investigating neuropathic or inflammatory pain (the interested reader is referred to these excellent reviews on imaging pain in the literature ${ }^{9-11}$ ).

Since 1906 we have known that the brain can modulate in a "top-down" manner spinal cord excitability via a tonically active influence that is largely inhibitory in function. Evidence to support this came from work by Sherrington who showed that nociceptive reflexes were enhanced after the spinal cord was transected..$^{12}$ Reynolds in 1969 again emphasised the relevance of this phenomenon by showing that focal electrical stimulation in the rat midbrain periaqueductal gray (PAG) produced analgesia strong enough to permit surgery. ${ }^{13}$ Over the years, further work showed that stimulation of several brain sites, including the sensory cortex, thalamus, hypothalamus, midbrain, pons, and medulla, produced inhibitory effects on spinal nociceptive processing, suggesting an integrated network of brain regions that produce anti-nociceptive influences in situations where it is desirable to not be behaviourally diverted due to the noxious input. Such situations could include those where there is high arousal as in sports or battle or during placebo analgesia. Many electrophysiological, anatomical, and pharmacological studies determined that these descending influences on spinal nociceptive processing relied on relays in the rostroventral medulla (RVM), including the medial nucleus raphe magnus and it is now accepted that the RVM is the final common output for descending influences from rostral brain sites (for an excellent review see Gebhart $2004^{14}$ and Porreca et al, 2002 ${ }^{15}$ ).

The focus of research on these descending influences was on inhibitory anti-nociceptive effects, with surgical applications for the treatment of chronic pain using periventricular electrical stimulation. $^{16}$ In the 1990s, pioneering work by Gebhart and colleagues established without doubt the additional presence of descending facilitatory influences on spinal nociceptive processing. Independent work led by Fields et al had focused on characterising the response properties of cells within the RVM and showed that two major types of neurones were present: "ON" and "OFF" ${ }^{17}$ This work by Fields et al paved the way for understanding better the role of the RVM in relation to processing and "top-down" modulation of pain. "OFF" cells are thought to comprise a descending inhibitory system that attenuates nociceptive information directly at the level of the spinal cord (anti-nociception) $)^{17} 18$ whereas "ON" cells have a facilitatory influence on nociceptive processing through descending systems projecting to the spinal cord (pro-nociceptive). ${ }^{17}$ The functional role of anti-nociception in everyday life and its importance is relatively easy to grasp; stress, fear, intense exercise, or escaping from a predator when injured are a few situations where control of pain has an obvious value to the behaving animal. However, to understand the functional role of pro-nociception in everyday life is less easy. Again, pioneering work by Gebhart and other colleagues unequivocally established a role for the RVM in the maintenance of hyperalgesic states following peripheral nerve injury. When tissue is injured there is increased input and sensitivity to stimulation at the site of injury; this is called primary hyperalgesia and is caused by increased excitability of peripheral nociceptors. More importantly, there is also increased sensitivity to stimuli from uninjured tissue either surrounding or distal to the site of injury; this is called secondary hyperalgesia and is caused by changes in excitability of neurones in the CNS or "central sensitisation". Most work has focused on spinal cord changes during generation of central sensitisation but the work described above on pro-nociceptive influences provided an alternative explanation for the generation and maintenance of secondary hyperalgesia. Work from the laboratories of Gebhart, Porreca, and others have clearly established the importance of such "spinobulbo-spinal" loops in hyperalgesia and possibly the development of chronic pain. ${ }^{14} 1519$ Indeed, recent work from our laboratory using FMRI in a human 
model of somatic secondary hyperalgesia has shown for the first time brainstem involvement in the generation and maintenance of hyperalgesia in humans. ${ }^{20}$ Many animal studies have now been published that unequivocally show that pro-nociception via the RVM plays a key role in generating and maintaining a cardinal symptom of chronic pain; hyperalgesia. Why? One obvious answer is to prevent further damage to an already damaged area of the body. However, there are numerous other examples of "functional disorders" (for example, irritable bowel syndrome, fibromyalgia, etc) that are associated with discomfort and pain but where tissue pathology is often lacking. What role pro-nociceptive influences have in these conditions, and the potential of higher brain centres influencing RVM pro-nociceptive output, has yet to be thoroughly established. One thing is certain however, which is that the enormous potential pro-nociceptive influences may play in human disease is only just being realised.

Concurrent with these developments in our understanding of spino-bulbospinal loops for generating and maintaining exaggerated pain behaviours in chronic pain conditions (that is, pain making the pain worse via RVM pronociceptive influences) has been observations of a different phenomenon in which a spino-bulbo-spinal loop produced analgesia after acute simultaneous noxious input (pain makes less pain): this phenomenon is called diffuse noxious inhibitory control (DNIC) and is commonly known as counterirritation. ${ }^{21}$ This phenomenon goes back to the Hippocratic aphorism: "If two sufferings take place at the same time, but at different points, the stronger makes the weaker silent". Experimental results during the 1980-early 1990s showed that counterirritation or counterstimulation has a well defined neural substrate in animals and humans. The mechanism to account for this phenomenon, DNIC, relies on spino-bulbospinal loops involving ascending pathways in the anterolateral spinal columns, integration in the lower brain stem, and descending influences or antinociception reaching the dorsal horn neurones. Neurones in the dorsal horn of the spinal cord were found to be inhibited when a nociceptive stimulus is applied to any part of the body that is distinct from the excitatory receptive fields of the dorsal horn neurones that are inhibited (hence the origin of the term "diffuse" as opposed to the similarly observed hypoalgesic effects of painful stimulation to the same segmental region of the body, as for instance during transcutaneous electrical nerve stimulation). DNIC generated inhibitions on dorsal horn neurones are potent and affect all the activities of the convergent neurones and persist after removal of the conditioning stimulus. This "pain inhibiting pain" effect is well known from folk medicine across many cultures. Indeed, in early surgical procedures on humans and animals this concept was harnessed without realisation of the underlying mechanisms (for example, use of the twitch in horses and nasal forceps in cattle during caudectomies or castrations which are both potentially very painful procedures). Reproducing this phenomenon in the human or animal laboratory has generated an enormous literature, and one simple example shows that ischaemic pain in the arm elevates the threshold for heat pain on the forehead and the threshold for painful stimulation of the tooth pulp. ${ }^{22}$ Many stressful stimuli are able to produce such counterirritation which has led to the term "stress induced analgesia". Studies have used ischaemic pain, cold pressor pain, noxious heat, and even long distance running as conditioning stimuli. ${ }^{22-25}$ Several surgical, pharmacological, and electrophysiological experiments on DNIC support the idea there is a spino-bulbo-spinal loop with minimal input from the PAGRVM or other supraspinal pathways. ${ }^{21}{ }^{26}$ This implies that a system exists distinct from the anti- and pro-nociceptive systems described above, possibly within more caudal regions of the brainstem, and this system is what drives DNIC. ${ }^{27} 28$

To date, these significant changes of phasic pain perception have been found by various psychophysical techniques, human reflex studies, and pain related evoked cerebral potentials ${ }^{29}$ but none has been reported using brain imaging methodologies in combination with visceral stimulation.

The work reported by Wilder-Smith and colleagues $^{30}$ in this issue of Gut extends current observations considerably by combining brain functional magnetic resonance imaging (FMRI) with DNIC and rectal balloon distension in IBS patient subgroups and healthy controls (see page 1595). They show that compared with rectal balloon stimulation alone, the median rectal pain scores to balloon distension in healthy controls during heterotopic stimulation (foot with ice water) were decreased significantly. This robust psychophysical finding was not found to be the case in IBS patients or subgroups. This psychophysical observation implies dysfunction of the systems subserving DNIC and has been shown in a previous study on fibromyalgia patients. ${ }^{31}$ It is interesting to note that these two different patient populations (IBS and fibromyalgia) that are notoriously difficult when it comes to finding a "cause" of the pain, display an apparent common dysfunction of DNIC mechanisms. The study by Wilder-Smith and colleagues, ${ }^{30}$ however, extends the observations to include functional brain imaging data, using FMRI, as a way of possibly explaining these psychophysical observations. They observed clear differences in brain activation patterns between controls and patient subgroups to rectal balloon distension both with and without heterotopic stimulation, highlighting an additional change in brain activation due to the dysfunctional DNIC system in patients. Combining brain imaging in patients with psychophysical manipulations provides a powerful approach for increasing our understanding of the brainstem structures involved in producing a DNIC effect. In addition, it has the capability of identifying higher cortical regions linked to such brainstem structures that as a consequence of disease might play a role in disabling the DNIC system. Their study has not provided all the answers, but neither should we expect it to, as many further experiments are required to establish the precise location and role of such brainstem and cortical regions. However, their study is the first of its kind and paves the way towards a better understanding of DNIC and how this acute experimental manipulation with its long history integrates with the above mentioned current theories of anti- and pro- nociception in the generation of hyperalgesia and chronic pain.

Work from our laboratory using counterstimulation to a noxious stimulus in conjunction with human FMRI showed that attention does play a major factor in the perceptual modulation of pain produced by counterstimulation. ${ }^{32}$ However, there are several studies suggesting that distraction does not account completely for the analgesia produced by activation of DNIC mechanisms, ${ }^{24} 262933$ including a fairly recent electrophysiological study in humans that supports the suggestion that the analgesic effect of heterotopic noxious stimulation in humans is based on activation of a specific inhibitory pain control system where release of endogenous opioids via the PAG-RVM system is unlikely to be involved..$^{29}$ The reciprocal issue as to whether hypervigilance to the acute pain stimulus during heterotopic stimulation causes disruption of DNIC effects has not been addressed, particularly in patients where the acute pain stimulus has a potential meaning, for instance rectal balloon distension in IBS patients. Of course, brain regions involved in 
maintaining a hypervigilant state could well be connected to brainstem structures involved in the DNIC system, and therefore this apparent confound could be the precise trigger for DNIC dysfunction. Again, this is speculation and further work is needed.

It is interesting to note that many of the changes Wilder-Smith and colleagues ${ }^{30}$ observed were in brain regions involved in controlling emotional, autonomic, and classic PAG descending pain modulatory systems, suggesting involvement of the PAG-RVM system. It is also possible that brainstem pro-nociceptive plastic changes have occurred in these IBS patient subgroups as part of the evolution towards chronicity and it is this change that contributes partly to their heightened sensitivity and pain report. This would fit with current theories of pro-nociception. ${ }^{19}$ Therefore, when acute experimental manipulations are subsequently performed on such patients to trigger DNIC effects, it could be that possible raised pro-nociceptive influences nullify or counteract any descending pain modulatory effects normally produced by DNIC, thereby accounting for a lack of behavioural pain relief. This is speculation and clearly further work must be done to further interrogate these possibilities. However, the study by Wilder-Smith and colleagues $^{30}$ does provide a basis for generating hypotheses that can be tested in such future experiments. This will ultimately lead to a fuller understanding of the pain component of IBS patients and hopefully better targeted treatments.

Gut 2004;53:1553-1555.

doi: $10.1136 /$ gut.2004.046110

n....................

Authors' affiliations

I Tracey, P Dunckley, Pain Imaging

Neuroscience (PalN) Group, Human Anatomy and Genetics Department and FMRIB Centre, Oxford University, Oxford, UK

Correspondence to: Dr I Tracey, Pain Imaging Neuroscience (PalN) Group, Human Anatomy and Genetics Department and FMRIB Centre, Oxford University, South Parks Rd, Oxford OXI 3QX, UK; irene.tracey@anat.ox.ac.uk

\section{REFERENCES}

1 Harstall C. How prevalent is chronic pain? Pain Clin Updates 2003;XI:1-4.

2 Ploghaus A, Narain C, Beckmann CF, et al. Exacerbation of pain by anxiety is associated with activity in a hippocampal network. J Neurosci 2001;21:9896-903.

3 Bantick SJ, Wise RG, Ploghaus A, et al. Imaging: how attention modulates pain in humans using functional MRI. Brain 2002;125:310-19.

4 Tracey I, Ploghaus A, Gati JS, et al. Imaging attentional modulation of pain in the periaqueductal gray in humans. $J$ Neurosci 2002;22:2748-52.

5 Aziz Q, Andersson JL, Valind S, et al. Identification of human brain loci processing esophageal sensation using positron emission tomography. Gastroenterology 1997; 1 13:50-9.

6 Silverman DH, Munakata JA, Ennes H, et al. Regional cerebral activity in normal and pathological perception of visceral pain. Gastroenterology 1997; 112:64-72.

7 Chang L, Berman S, Mayer EA, et al. Brain responses to visceral and somatic stimuli in patients with irritable bowel syndrome with and without fibromyalgia. Am J Gastroenterol 2003:98:1354-61

8 Wall P, Melzack R. Textbook of pain, 4th edn. Edinburgh: Churchill Livingstone, 1999.

9 Treede RD, Kenshalo DR, Gracely RH, et al. The cortical representation of pain. Pain 1999;79:105-11.

10 Peyron R, Laurent B, Garcia-Larrea L. Functional imaging of brain responses to pain. A review and meta-analysis. Neurophysiol Clin 2000;30:263-88

11 Craig AD. How do you feel? Interoception: the sense of the physiological condition of the body. Nat Rev Neurosci 2002;3:655-66.

12 Sherrington $\mathrm{C}$. The integrative action of the nervous system. Oxford: Oxford University Press, 1906.

13 Reynolds DV. Surgery in the rat during electrical analgesia induced by focal brain stimulation. Science 1969;164:444-5.

14 Gebhart GF. Descending modulation of pain Neurosci Biobehav Rev 2004;27:729-37.

15 Porreca F, Ossipov MH, Gebhart GF. Chronic pain and medullary descending facilitation. Trends Neurosci 2002;25:319-25.

16 Baskin DS, Mehler WR, Hosobuchi Y, et al Autopsy analysis of the safety, efficacy and cartography of electrical stimulation of the central gray in humans. Brain Res 1986:371:231-6.

17 Fields HL, Bry J, Hentall I, et al. The activity of neurons in the rostral medulla of the rat during withdrawal from noxious heat. J Neurosci 1983;3:2545-52.

18 Fields HL, Heinricher MM. Anatomy and physiology of a nociceptive modulatory system.
Philos Trans $R$ Soc Lond B Biol Sci 1985;308:361-74

19 Urban MO, Gebhart GF. Supraspinal contributions to hyperalgesia. Proc Natl Acad Sci U S A 1999;96:7687-92

20 Zambraneau L, Wise R, Brooks J, et al. FMRI evidence for midbrain reticular formation involvement in a human model of neuropathic pain. J Pain 2004;5(suppl 1):310 (abstract).

21 Villanueva $L$, Le Bars $D$. The activation of bulbo spinal controls by peripheral nociceptive inputs: diffuse noxious inhibitory controls. Biol Res 1995;28:113-25.

22 Pertovaara A, Kemppainen P, Johansson G, et al. Ischemic pain nonsegmentally produces a predominant reduction of pain and thermal sensitivity in man: a selective role for endogenous opioids. Brain Res 1982;251:83-92.

23 Talbot JD, Duncan GH, Bushnell MC, et al. Ischemic pain nonsegmentally produces a predominant reduction of pain and thermal sensitivity in man: a selective role for endogenous opioids. Brain Res 1982;251:83-92.

24 Willer JC, De Broucker T, Le Bars D. Encoding of nociceptive thermal stimuli by diffuse noxious inhibitory controls in humans. J Neurophysiol 1989;62:1028-38.

25 Janal MN, Colt EW, Clark WC, et al. Pain sensitivity, mood and plasma endocrine levels in man following long-distance running: effects of naloxone. Pain 1984;19:13-25.

26 Willer JC, Bouhassira D, Le Bars D. Neurophysiological bases of the counterirritation phenomenon: diffuse control inhibitors induced by nociceptive stimulation. Neurophysiol Clin 1999;29:379-400.

27 Fields HL, Basbaum AJ. Endogenous pain control mechanisms. In: Wall PD, Melzack R, eds. Textbook of pain. Edinburgh: Churchill Livingstone, 1989:206-17.

28 Le Bars D, Bouhassira D, Villanueva L. Opioids and diffuse noxious inhibitory control (DNIC) in the rat. In: Bromm B, Desmedt JE, eds. Advances in pain research and therapy. New York: Raven Press, 1995:517-39.

29 Reinert A, Treede R, Bromm B. The pain inhibiting pain effect: an electrophysiological study in humans. Brain Res 2000;862:103-10.

30 Wilder-Smith C, Schindler D, Lovblad K, et al. Brain functional magnetic resonance imaging of rectal pain and activation of endogenous inhibitory mechanisms in irritable bowel syndrome patient subgroups and healthy controls. Gut 2004;53:1595-601.

31 Kosek E, Hansson P. Modulatory influence on somatosensory perception from vibration and heterotopic noxious conditioning stimulation (HNCS) in fibromyalgia patients and healthy subjects. Pain 1997;70:41-51

32 Longe SE, Wise R, Bantick S, et al. Counterstimulatory effects on pain perception and processing are significantly altered by attention: an FMRI study. Neuroreport 2001;12:2021-5.

33 Kakigi R. Diffuse noxious inhibitory controls. J Neurol Sci 1994;125:198-205. 
T cell apoptosis

\section{T cell apoptosis and inflammatory bowel disease}

\section{P Peppelenbosch, S J H van Deventer}

Intrinsic defects in the control of programmed cell death in the mucosal T cell compartment are strongly implicated in the pathogenesis of inflammatory bowel diseases, and there are important differences in the regulation of apoptosis between ulcerative colitis and Crohn's disease

A pproximately $2 \times 10^{14}$ bacteria reside in the gastrointestinal tract, and this astonishing microbiological pressure represents an extraordinary challenge to the mucosal immune system, which has to perform a balancing act between appropriate responsiveness to pathogenic organisms and tolerance for harmless organisms. When this balance in mucosal immune system responsiveness is tilted towards an exaggerated or uncontrolled reaction against the commensal flora, inflammatory bowel disease results. Despite many important findings in recent years, including the association of inactivating mutations in the CARD15 (NOD2) receptor with Crohn's disease, it is not fully understood why the mucosal immune response is over reactive in patients with inflammatory bowel disease, or how current medical treatment precisely affects the mucosal immune system. Two papers in the present issue of $G u t^{12}$ suggest that intrinsic defects in the control of programmed cell death in the mucosal $\mathrm{T}$ cell compartment are strongly implicated in the pathogenesis of inflammatory bowel diseases, and important differences in the regulation of apoptosis between ulcerative colitis and Crohn's disease are identified (see pages 1624 and 1632 ).

The experiments performed in both papers compare properties of apoptosis induction in lamina propria $\mathrm{T}$ cells of patients of Crohn's disease, ulcerative colitis, and non-inflamed controls. The paper by Doering and colleagues ${ }^{1}$ reports that induction of apoptosis in mucosal $\mathrm{T}$ cell compartment is severely impaired in both Crohn's disease and ulcerative colitis. This is the latest of a series of observations by several laboratories which show that mucosal $\mathrm{T}$ cells of patients with inflammatory bowel disease are highly resistant to apoptosis. ${ }^{3-6}$ Because apoptosis of activated $\mathrm{T}$ cells is an important mechanism of peripheral immune tolerance, this finding may explain why the inflammatory reaction in IBD patients is resilient to resolution. Apoptosis is regulated by two major intracellular pathways. Activation of the Fas receptor leads to recruitment and activation of caspase 8 , which activates caspase 3, leading to a cascade of downstream events (DNA cleavage, cell membrane changes, etc), finally causing programmed cell death. Caspase 3 can also be activated by a complex of proteins, including caspase 9, APAF-1, and cytochrome $\mathrm{C}$, know as the apoptosome. This pathway is activated by leakage of cytochrome $\mathrm{C}$ from the mitochondria, which is regulated by the Bcl-2 family of proteins (see fig l). The apoptosis defect in mucosal T cells in Crohn's disease has been attributed to an imbalance of the antiapoptotic mitochondrial protein $\mathrm{Bcl}-2$ and the proapoptotic mitochondrial protein Bax. $^{35}$ In agreement, in the study of Sturm and colleagues ${ }^{2}$ in the current issue of Gut, no decreased caspase 8 activity (which acts upstream of both the mitochondrial and non-mitochondrial pathway of apoptosis ${ }^{7}$ ) or increased expression of the caspase 8 inhibitor FLIP were observed, but caspase 3 activity (which is downstream of the mitochondria in induction of apoptosis $^{7}$ ) was reduced in the mucosal $\mathrm{T}$ cells of patients with Crohn's disease (see fig 1). Hence the available data are consistent with the notion that the apoptosis defect of mucosal $\mathrm{T}$ cells in Crohn's disease occurs at the level of the mitochondria in the mitochondrial pathway to programmed cell death.

This apoptosis defect in the mitochondrial pathway has important implications for the treatment of Crohn's disease. Van den Brande et al have shown that the difference in effectiveness of tumour necrosis factor neutralising drugs for treating Crohn's disease is reflected in their capacity to induce apoptosis of activated $\mathrm{T}$ cells. ${ }^{8}$ Infliximab is highly effective in Crohn's disease ${ }^{9}$ and is a strong inducer of apoptosis in lamina propria $\mathrm{T}$ cells ${ }^{10}$ whereas etanercept offers no clinical benefit in Crohn's disease ${ }^{11}$ and does not have the capacity to induce apoptosis of these cells. ${ }^{8}$ In the paper of Doering and colleagues ${ }^{1}$ in the present issue of Gut, these observations are further extended by showing that sulphasalazine is also a potent inducer of apoptosis in $\mathrm{T}$ cells. The sulphasalazine effect was accompanied by downregulation of the antiapoptotic mitochondrial proteins $\mathrm{Bcl}-\mathrm{xl}$ and $\mathrm{Bcl}-2$, again in agreement with an apoptosis defect at the level of the mitochondria in the mucosal $\mathrm{T}$ cells of Crohn's disease patients. It should be kept in mind that within the colon, sulphasalazine is cleaved by bacterial enzymes to yield sulphapyridine and 5-aminosalicylic acid. It is commonly thought that the therapeutic efficacy of sulphasalazine is mediated by 5-aminosalicylic acid, sulphapyridine being responsible for sulphasalazine related side effects. However, the paper by Doering and colleagues $^{1}$ indicates that the effects of mesalazine and sulphasalazine on activated $\mathrm{T}$ cells are very different, and because the clinical studies that lead to the notion that mesalazine is the therapeutic moiety within sulphasalazine were conducted in patients suffering from ulcerative colitis, and not in Crohn's disease patients, it is possible that an important therapeutic effect of sulphasalazine has been overlooked. It will be important to know how much uncleaved sulphasalazine remains in the colon, and what fraction of uncleaved sulphasalazine enters the intestinal mucosa.

Two other drugs, the antimetabolites azathioprine/6-mercaptopurine and methotrexate, have been reported to also induce apoptosis of activated $\mathrm{T}$ cells. For azathioprine/6-mercaptopurine the mechanism of apoptosis induction has been recently linked to inhibition of CD28 signalling by the 6-mercaptopurine metabolite 6-thioguanine-triphosphate. ${ }^{12}$ Because activation of the costimulatory molecule CD28 protects $\mathrm{T}$ cells against apoptosis, this finding suggests that the therapeutic effect of azathioprine/6-mercaptopurine may also be related to induction of apoptosis of activated $\mathrm{T}$ cells. Indeed, CD3/CD28 stimulated $\mathrm{T}$ cells undergo apoptosis when exposed to azathioprine or 6-mercaptopurine. Because the molecular apoptotic pathways that are affected by infliximab, azathioprine, methotrexate, and sulphasalazine are likely to be different, these drugs may have additional or even synergistic 
A

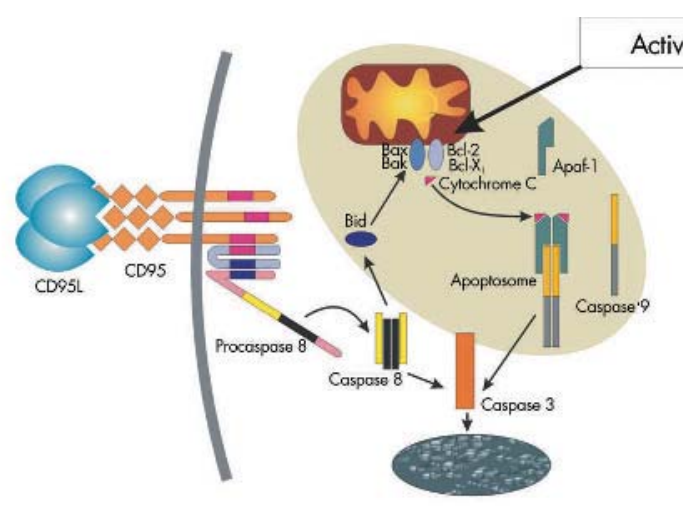

B

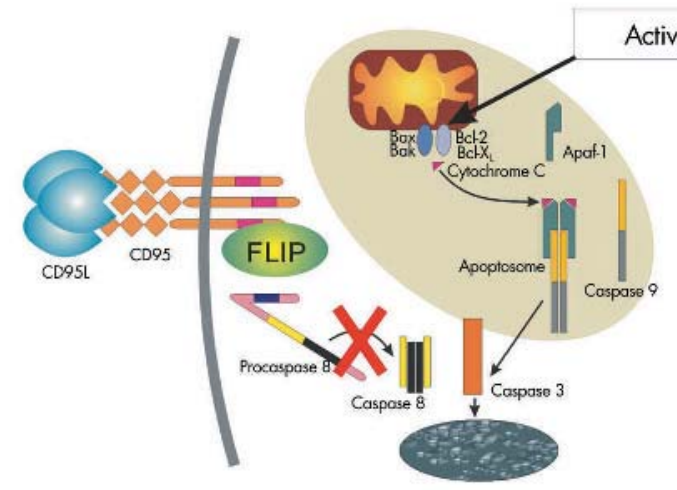

c

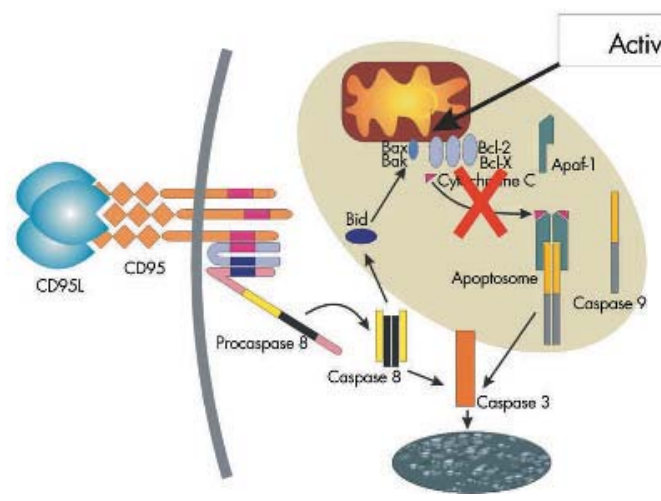

Figure 1 Two major intracellular pathways cause apoptosis. (A) Binding of FasL to CD95 (Fas receptor) leads to recruitment and activation of procaspase 8 , which subsequently activates caspase 3. Activated caspase 3 is responsible for induction of several events that lead to apoptosis. A second apoptotic pathway is initiated by leakage of cytochrome $C$ from the mitochondria, which together with caspase 9 and APAF1 forms the apoptosome that can activate caspase 3 . The integrity of the mitochondrial membrane is regulated by proteins of the Bcl-2 family, Bax and Bak being proapoptotic, $\mathrm{Bcl}-2$ and $\mathrm{Bcl}-\mathrm{xl}$ antiapoptotic. (B) Apoptosis resistance in ulcerative colitis results from overexpression of FLIP, leading to impairment of the caspase mediated pathway of apoptosis. The mitochondrial pathway is intact. (C) In contrast, in Crohn's disease, Fas induced apoptosis is normal but the mitochondrial pathway is affected by an imbalance of pro- and antiapoptotic Bcl-2 family members. As a consequence, activation induced apoptosis of $\mathrm{T}$ cells is impaired.

therapeutic effects. It is of the utmost importance that this possibility be studied in future clinical trials.

In accordance with earlier observations, ${ }^{3}$ Doering et al report that lamina propria lymphocytes isolated from patients with ulcerative colitis were also more resistant to Fas ligand induced apoptosis. ${ }^{1}$ This apoptosis resistance in ulcerative colitis is clearly different from that observed in Crohn's disease because lamina propria $\mathrm{T}$ cells from ulcerative colitis patients display increased apoptosis or caspase activation on $\mathrm{CD} 2$ or $\mathrm{CD} 3$ ligation, suggesting that the resistance to apoptosis through the Fas pathway is not reflected in a defect in activation induced apoptosis in the mucosal $\mathrm{T}$ cell compartment of these patients. Importantly, Fas ligand mediated apoptosis is mediated through activation of caspase 8 and Sturm and colleagues $^{2}$ show that ulcerative colitis patients have significantly higher expression of the caspase 8 inhibitor FLIP, suggesting that resistance to apoptosis in this disease is mediated at this level. Thus regulation of programmed cell death seems fundamentally different in mucosal $\mathrm{T}$ cells from patients with Crohn's disease compared with patients with colitis ulcerosa, and probably requires a different therapeutic strategy.

The latter notion is substantiated by the observations of Sturm and colleagues $^{2}$ in their comparison of the cell cycle properties of lamina propria lymphocytes from patients with Crohn's disease and ulcerative colitis. Using various parameters it is shown that $\mathrm{T}$ lymphocytes of patients with Crohn's disease cycle faster and have an impressive capacity for cellular expansion when compared with mucosal $\mathrm{T}$ cells of inflamed controls, probably as a result of a defect in activation dependent apoptosis. The abnormally rapid cycling of lamina propria $\mathrm{T}$ cells is also very different from several other immune mediated diseases that are characterized by a $G_{0} / G_{1}$ cell cycle arrest of activated $T$ cells. ${ }^{13}{ }^{14}$ Such $G_{0} / G_{1}$ arrested cells will not undergo apoptosis, and in experimental SLE, induction of $\mathrm{T}$ cell cycling leads to progression of $\mathrm{T}$ cells into apoptosis and has remarkable therapeutic effects. ${ }^{15}$ Hence although it has become popular to speculate about common mechanisms that lead to immune mediated inflammatory diseases, these observations clearly demonstrate fundamentally different defects that all may lead to impaired $\mathrm{T}$ cell apoptosis. These differences are not only important for our understanding of the pathogenesis of these diseases but also for the development of new therapies. For example, it would not be a good idea to try to boost apoptosis of lamina propria $\mathrm{T}$ cells in Crohn's disease by induction of cell cycling.

$\mathrm{T}$ cells from ulcerative colitis patients have a limited capacity to expand and this correlates with a strong activation induced apoptosis in these cells. This observation dramatically shows that the defect in the mitochondrial pathway at the mitochondrial level mediates the reduced activation induced cell death in Crohn's disease whereas inhibition of Fas dependent apoptosis by increased expression of the caspase 8 inhibitor FLIP (that is, upstream of the mitochondria in the apoptotic cascade) in ulcerative colitis does not result in inhibition of activation induced cell death. These 
molecular differences in apoptosis inhibition between Crohn's disease and ulcerative colitis strongly indicate that the underlying immune mechanisms are distinct. Furthermore, these observations correspond well with emerging insight that peripheral deletion of $\mathrm{T}$ lymphocytes by activation induced cell death is not mediated by Fas activation but by regulation of expression of the mitochondrial proapoptotic bcl-2 family member bim. ${ }^{16}{ }^{17}$ As resistance to Fas ligand induced apoptosis in mucosal $\mathrm{T}$ lymphocytes seems to be mediated at the level of FLICE inhibition (which acts upstream of the mitochondria), peripheral activation induced cell death in colitis ulcerosa is not impaired.

Hence the papers of Strum et al and Doering et al in the present issue of Gut give substantial novel molecular insights into regulation of apoptosis in the mucosal $\mathrm{T}$ cell compartment and provide further support for the concepts that (1) the defect in apoptosis in mucosal $\mathrm{T}$ cells is an important contributing factor in the pathogenesis of Crohn's disease but not colitis ulcerosa, and (2) that restoration of apoptosis in the $\mathrm{T}$ mucosal $\mathrm{T}$ cell compartment may be an important contributing factor for successful treatment of Crohn's disease.

Gut 2004;53:1556-1558.

doi: $10.1136 /$ gut.2004.040824

\section{Authors' affiliations}

M P Peppelenbosch, S J H van Deventer,

Department of Gastroenterology and

Hepatology, Academic Medical Centre,

Amsterdam, the Netherlands

Correspondence to: Dr S J H van Deventer, Department of Gastroenterology and Hepatology, C2-330, Academic Medical Centre, Meibergdreef 9, NL-1105 AZ Amsterdam, the Netherlands; s.j.vandeventer@ amc.uva.nl

\section{REFERENCES}

1 Doering J, Begue B, Lentze MJ, et al. Induction of $T$ lymphocyte apoptosis by sulphasalazine in patients with Crohn's disease. Gut 2004;53:1632-8.

2 Sturm A, Leite AZ, Danese S, et al. Divergent cell cycle kinetics underlie the distinct functional capacity of mucosal T cells in Crohn's disease and ulcerative colitis. Gut 2004;53:1624-31.

3 Ina K, Itoh J, Fukushima K, et al. Resistance of Crohn's disease T cells to multiple apoptotic signals is associated with a Bcl-2/Bax mucosal imbalance. J Immunol 1999;163:1081-90.

4 Boirivant M, Marini M, Di Felice G, et al. Lamina propria T cells in Crohn's disease and other gastrointestinal inflammation show defective CD2 pathway-induced apoptosis. Gastroenterology 1999; 1 16:557-65.

5 Itoh J, de La Motte C, Strong SA, et al. Decreased Bax expression by mucosal T cells favours resistance to apoptosis in Crohn's disease. Gut 2001;49:35-41.

6 Atreya R, Mudter J, Finotto S, et al. Blockade of interleukin 6 trans signaling suppresses T-cell resistance against apoptosis in chronic intestinal inflammation: evidence in Crohn disease and experimental colitis in vivo. Nat Med 2000;6:583-8.

7 Festiens N, van Gurp M, van Loo G, et al. Bcl-2 family members as sentinels of cellular integrity and role of mitochondrial intermembrane space proteins in apoptotic cell death. Acta Haematol 2004;111:7-27.

8 Van den Brande JM, Braat $H$, van den Brink GR, et al. Infliximab but not etanercept induces apoptosis in lamina propria T-lymphocytes from patients with Crohn's disease. Gastroenterology 2003; 124:1774-85.

9 Targan SR, Hanauer SB, van Deventer SJ, et al. A short-term study of chimeric monoclonal antibody cA2 to tumor necrosis factor alpha for Crohn's disease. Crohn's Disease cA2 Study Group. N Engl J Med 1997;337:1029-35.

10 ten Hove T, van Montfrans C, Peppelenbosch MP, et al. Infliximab treatment induces apoptosis of lamina propria T lymphocytes in Crohn's disease. Gut 2002;50:206-11.

11 Sandborn WJ, Hanauer SB, Katz S, et al. Etanercept for active Crohn's disease: a randomized, double-blind, placebo-controlled trial. Gastroenterology 2001;121:1088-94.

12 Tiede I, Fritz G, Strand S, et al. CD28-dependent Racl activation is the molecular target of azathioprine in primary human $\mathrm{CD} 4+\mathrm{T}$ lymphocytes. J Clin Invest 2003;111:1133-45.

13 Ponchel F, Morgan AW, Bingham SJ, et al. Dysregulated lymphocyte proliferation and differentiation in patients with rheumatoid arthritis. Blood 2002;100:4550-6.

14 Henriksson G, Brant M, Sallmyr A, et al. Enhanced DNA damage-induced p53 peptide phosphorylation and cell-cycle arrest in Sjogren's syndrome cells. Eur J Clin Invest 2002;32:458-65.

15 Lawson BR, Baccala R, Song J, et al. Deficiency of the cyclin kinase inhibitor p21(WAF-1/CIP-1) promotes apoptosis of activated/memory T cells and inhibits spontaneous systemic autoimmunity. J Exp Med 2004; 199:547-57.

16 Hildeman DA, Zhu Y, Mitchell TC, et al. Activated $\mathrm{T}$ cell death in vivo mediated by proapoptotic bcl2 family member bim. Immunity 2002;16:759-67.

17 Davey GM, Kurts C, Miller JF, et al. Peripheral deletion of autoreactive CD8 T cells by cross presentation of self-antigen occurs by a Bcl-2inhibitable pathway mediated by Bim. J Exp Med 2002; 196:947-55

\section{NOD2 mutations and Crohn's disease: are Paneth cells and their antimicrobial peptides the link?}

\section{C Grimm, P Pavli}

There is evidence that CARD15/NOD2 variants in Crohn's disease are associated with diminished mucosal $\alpha$-defensin production, resulting in impaired innate immunity

\section{PANETH CELLS AND DEFENSINS}

Paneth cells (reviewed by Cunliffe and colleagues $^{12}$ ) are granulated epithelial cells found at the base of small intestinal crypts. They are most numerous in the terminal ileum and are not found in the normal colon. They secrete a variety of antimicrobial peptides and proteins, including $\alpha$-defensins, lysozyme, and secretory phospholipase $\mathrm{A}_{2}\left(\mathrm{sPLA}_{2}\right)$, in response to bacterial products and cholinergic agents. There is a link between intestinal inflammation and Paneth cell number. In mouse models of enteric infection, Paneth cell hyperplasia occurs in response to signals from activated $\mathrm{T}$ cells, in mouse models of ileocolitis there is Paneth cell metaplasia, and in inflammatory bowel disease, Paneth cell metaplasia is also seen in the colon. Parasite infestations also induce an increase in the number of Paneth cells in the murine small intestine.

Defensins (reviewed by $\mathrm{Ganz}^{3}$ ) are a family of small (29-45 amino acid) cationic antimicrobial peptides that are divided into two main classes, the $\alpha$ and $\beta$-defensins, on the basis of their disulphide bond pairing pattern. They are encoded by single genes as prepropeptides, which are then processed to mature active forms. The mammalian defensin genes probably evolved from a single ancestral gene but because of selection pressure and rapid evolution, comparisons between species is difficult. ${ }^{4}$

Six $\alpha$-defensins have been identified in humans: four are neutrophil peptides and two human defensins (HD-5 and HD-6) are expressed in intestinal Paneth cells. HD-5 is expressed in Paneth cells of the duodenum, jejunum, and ileum, but is not found in the normal stomach or colon. In cases of gastritis, Crohn's colitis, and ulcerative colitis, HD-5 is expressed in metaplastic Paneth cells.

In vitro, $\alpha$-defensins have broad spectrum antimicrobial activity and are active against a variety of bacteria, fungi, parasites, and viruses. Neutrophil 
defensins kill bacteria engulfed in phagolysosomes by formation of microbial selective, membrane spanning pores which lead to cell lysis.

In contrast with enteric $\alpha$-defensins, human $\beta$-defensin 1 (HBDl) and other members of the $\beta$-defensin family appear to be expressed by most epithelial cells of the small and large intestine. HBD- 1 is expressed constitutively while HBD-2 is induced by stimuli that activate the transcription factor nuclear factor $\kappa \mathrm{B}(\mathrm{NF} \kappa \mathrm{B}) .^{5}$

\section{TISSUE DISTRIBUTION OF CARD 15/NOD2}

Hugot and colleagues ${ }^{6}$ and Ogura and colleagues $^{7}$ independently identified mutations in CARD15/NOD2 that confer susceptibility to Crohn's disease. The three main disease associated alleles $\left(\mathrm{Arg}_{702} \operatorname{Trp}(\mathrm{C} 2104 \mathrm{~T}), \mathrm{Gly}_{908} \mathrm{Arg}\right.$ (G2722C) and $1007_{\mathrm{fs}}$ (3020insC)) and other variants cosegregate with disease in up to $30-50 \%$ of patients, depending on the population studied.

The protein product of this gene is thought to be a cytoplasmic receptor for muramyl dipeptide, a breakdown product of the peptidoglycan component of all bacterial cell walls. ${ }^{8} 9$ Lysozyme, including that derived from Paneth cells, is involved in this process by breaking the $\alpha-1,4$ glycosidic linkages in the polysaccharide components of peptidoglycans. CARD15/NOD2 is thought to act through the NFKB pathway to regulate production of tumour necrosis factor (TNF)- $\alpha$ and other proinflammatory cytokines. ${ }^{10}$

Hisamatsu and colleagues ${ }^{11}$ showed that CARD15/NOD2 mRNA was expressed and CARD15/NOD2 protein was detectable in intestinal epithelial cells (IECs). Using stable transfectants, they showed that CARD15/NOD2 was a potent factor for recognition of intracellular bacteria in IECs and that this function was impaired in 3020insC variant CARD15/NOD2 expressing IECs.

Definitive studies of the distribution of CARD15/NOD2 in vivo showed expression in Paneth cells of the normal duodenum, jejunum, and terminal ileum (the greatest staining intensity was noted in terminal ileal crypts). In inflammatory bowel disease, CARD15/ NOD2 expression in Paneth cells was increased and was also evident in metaplastic Paneth cells in the colon. ${ }^{12}$

Genotype-phenotype studies show that the known CARD15/NOD2 variants are more likely to have terminal ileal involvement. ${ }^{13-15}$

These observations raised the possibility that there was a link between CARD15/NOD2 variants and defective $\alpha$-defensin release by Paneth cells, resulting in impaired innate immunity.
Paneth cells detect and respond to bacteria, and the CARD15/NOD2 protein may mediate their interaction with bacterial products. Inherited mutations in CARD15/NOD2 could interfere with normal Paneth cell function resulting in a disordered immune response.

A paper by Wehkamp and colleagues ${ }^{16}$ in this issue of Gut provides supportive evidence that CARD15/NOD2 variants in Crohn's disease are associated with diminished mucosal $\alpha$-defensin production (see page 1658). Wehkamp et al examined expression of HD-5, HD-6, lysozyme, sPLA 2 TNF, and IL-8 in 45 Crohn's disease patients (24 with CARD15/NOD2 variants and 21 without) and 12 controls. They found that expression of HD-5 and HD-6 specifically was diminished in affected ileum, particularly in those bearing CARD15/ NOD2 variants. Other proteins and cytokines were unaffected. In the colon, HD-5 and HD-6 expression was increased during inflammation in wildtype but not CARD15/NOD2 variant bearing patients. There are some unexplained results in this paper: why were TNF- $\alpha$ levels not increased in inflamed ileum? Why were lysozyme levels in unaffected wild-type ileum not the same as in controls? How do they explain the increase in $\alpha$-defensins in inflamed colon while levels were decreased in the inflamed small bowel?

\section{CARD 15/NOD2 LINKS PANETH CELL DEFENSINS TO CROHN'S DISEASE: FOR}

The predominant site of disease localisation in the CARD15/NOD2 variant bearing patients is the terminal ileum which is also the site of maximal Paneth cell density-guilt by association. The density of Peyer's patches is also greatest in the terminal ileum. These organised collections of lymphoid cells play a role in the selective uptake of luminal antigens. They are also the site of the earliest lesion in Crohn's disease-the aphthous ulcer, an area of focal ulceration over a Peyer's patch (in the small intestine) or lymphoid follicle (in the colon). Perhaps Paneth cell $\alpha$-defensins present in high levels in the terminal ileum inactivate luminal bacteria taken up by Peyer's patches or colonic lymphoid follicles so the immune system need only deal with inactivated bacteria rather than viable microorganisms.

In addition, $\alpha$-defensins (and their $\beta$ family counterparts) play a linking role between the innate immune system, characterised by rapid antimicrobial responses, and adaptive immunity instigated by $\mathrm{B}$ and $\mathrm{T}$ cells. The $\alpha$-defensins are selectively chemotactic for resting naïve $\mathrm{CD}^{+} \mathrm{T}$ cells and immature dendritic cells although at concentrations several logs lower than their antimicrobial concentrations. ${ }^{17}$ Impaired release of defensins from Paneth cells might thereby augment this chemotactic potential while simultaneously reducing their antipathogen effector function. Similarly, $\alpha$-defensins act as immunological adjuvants, heightening Thl and especially Th2 immune responses to nonspecific antigens ${ }^{18}$ and to tumours ${ }^{19}$; a reduction in mucosal defensin concentrations might allow skewing of the immune response to the Thl profile characteristic of Crohn's disease.

Most importantly, CARD15/NOD2 and $\alpha$-defensins potentially are linked in the Paneth cell as recognition and effector molecules, respectively, for pathogen associated molecular pattern (PAMP) motifs: their colocalisation in these cells is difficult to ignore. Some of these PAMPs, including lipopolysaccharide, lipoteichoic acid, dsRNA and, significantly, muramyl dipeptide, have been shown in an elegant study to induce rapid release of defensins from murine ileal crypt Paneth cells. ${ }^{20}$ Indeed, this defensin release occurred within 15 minutes and therefore did not reflect an effect on gene expression. While Wehkamp et al did show an association between defective CARD15/NOD2 and $\alpha$-defensin gene expression, a much more profound effect might yet be detected in secretion of stored $\alpha$-defensins. An analogous situation may have been observed, unknowingly, in intestinal epithelial cell lines which are able to produce $\beta$-defensins: when transfected with wild-type CARD15/NOD2, these cells resist infection with Salmonella typhimurium; if transfected with mutated CARD15/NOD2, they are more easily infected. ${ }^{11}$

While many of the specific details linking genetic defects with reduced antimicrobial resistance in Crohn's disease are still to be identified, there is strengthening circumstantial evidence to suggest that CARD15/NOD2 variant mediated deficiency of Paneth cell derived $\alpha$-defensins is critical in the development of some forms of Crohn's disease.

\section{CARD 15/NOD2 LINKS PANETH CELL DEFENSINS TO CROHN'S DISEASE: AGAINST}

Direct proof of the functional significance of these peptides in vivo is scarce. How important is a specific defensin when there are other antimicrobial molecules with similar properties? How important are Paneth cells? In one transgenic mouse strain, ablating the entire Paneth cell population had no observable effect on host-microbial interactions. ${ }^{21}$ Moreover, mice survive 
even though they lack granulocyte defensins completely.

However, there is evidence that defensin deficiency does result in impaired host resistance: knockout mice that cannot process the prepropeptides and secrete inactive cryptdin precursors are more susceptible to specific infections than wild-type littermates. Transgenic mice that express (human) HD-5 (as well as the normal crytpdins) in Paneth cells are resistant to oral infections compared with controls, indicating that HD-5 is an effective luminal antimicrobial. $^{22}$

Nevertheless, these and other studies of defensin deficiencies in experimental systems generally demonstrate susceptibility to specific infections rather than non-specific inflammation, in much the same way as selective IgA or other immunodeficiencies result in (generally) identifiable infections in humans. It is possible, as implied by Lala and colleagues, ${ }^{12}$ that in Paneth cells there is a Crohn's disease related dysregulation of TNF gene expression (and presumably protein production or secretion) but there is much to be done to prove this hypothesis. If defensin deficiencies result in increased susceptibility to infections (either specific or non-specific) we have to be able to explain the "effectiveness" of immunosuppressive medication in controlling symptoms of $\mathrm{CD}$-or at least not worsening the disease.

Much is made of the predominant terminal ileal involvement found in CARD15/NOD2 variant bearing patients. However, many of these patients have colonic involvement, generally in association with ileal disease, but sometimes exclusively. In the later stages of disease, there is Paneth cell metaplasia in the colon, but this is not present in the early phase. How does a defect of terminal ileal Paneth cell derived $\alpha$-defensins result in left sided colonic involvement? There are plausible explanations other than Paneth cell density. For example, the terminal ileum is also the site of greatest density and size of Peyer's patches; it may be the ability of the associated $M$ cells to take up luminal antigens and deliver them to the underlying lymphoid structures that initiates Crohn's disease. This same process may also induce aphthous ulceration over lymphoid follicles in the colon.

It may indeed be that there is a CARD15/NOD2 variant mediated deficiency of Paneth cell derived $\alpha$-defensins but there are still numerous steps that need to be taken before there is conclusive proof. For example, there are no NFKB binding sites in the promoter regions of the Paneth cell $\alpha$-defensins so the means by which CARD15/NOD2 variants affect $\alpha$-defensin protein production and secretion need to be defined. Obtaining the evidence will be very difficult in the absence of appropriate experimental systems to study signal transduction in Paneth cells.

According to Sherlock Holmes "...when you have eliminated the impossible, whatever remains... must be the truth." (The Sign of Four. Sir Arthur Conan Doyle). There is evidence implicating the Paneth cell but it is not beyond reasonable doubt. Also, there is another possibility: the newly arrived monocyte is known to express high levels of CARD15/NOD2 ${ }^{12}$ and to possess a plethora of antimicrobial and other effector functions. It remains a prime suspect and, like the Paneth cell, could also be regarded as guilty until proven innocent.

Gut 2004;53:1558-1560.

doi: $10.1136 /$ gut.2004.043307

\section{Authors' affiliations}

M C Grimm, Department of Medicine, St George Clinical School, University of NSW, Sydney, Australia

P Pavli, Gastroenterology Unit, Canberra

Hospital, Canberra, Australia

Correspondence to: Dr P Pavli,

Gastroenterology Unit, Canberra Hospital,

Woden ACT 2606, Canberra, Australia; paul.pavli@act.gov.au

\section{REFERENCES}

1 Cunliffe RN. $\alpha$-Defensins in the gastrointestinal tract. Mol Immunol 2003:40:463-7.

2 Cunliffe RN, Mahida YR. Expression and regulation of antimicrobial peptides in the gastrointestinal tract. J Leukoc Biol 2004;75:49-58.

3 Ganz T. Defensins: antimicrobial peptides of innate immunity. Nat Rev Immunol 2003;3:710-20.
4 Hughes AL. Evolutionary diversification of the mammalian defensins. Cell Mol Life Sci 1999:56:94-103.

5 O'Neil DA, Porter EM, Elewaut D, et al. Expression and regulation of the human b-defensins hBD-1 and hBD-2 in intestinal epithelium. J Immunol 1999; 163:6718-24.

6 Hugot J-P, Laurent-Puig P, Gower-Rousseau C, et al. Mapping of a susceptibility locus for Crohn's disease on chromosome 16. Nature 1996;379:821-3

7 Ogura $\mathrm{Y}$, Bonen DK, Inohara N, et al. A frameshift mutation in NOD2 associated with susceptibility to Crohn's disease. Nature 2001;411:603-6.

8 Inohara N, Ogura Y, Fontalba A, et al. Host recognition of bacterial muramyl dipeptide mediated through NOD2. Implications for Crohn's disease. J Biol Chem 2003;278:5509-12.

9 Girardin SE, Boneca IG, Viala J, et al. Nod2 is a general sensor of peptidoglycan through muramyl dipeptide (MDP) detection. J Biol Chem 2003;278:8869-72.

10 Ogura Y, Inohara N, Benito A, et al. Nod2, a Nod1/Apaf-1 family member that is restricted to monocytes and activates NF-kB. J Biol Chem 2001;276:4812-18.

11 Hisamatsu T, Suzuki M, Reinecker HC, et al. CARD 15/NOD2 functions as an antibacterial factor in human intestinal epithelial cells. Gastroenterology 2003;124:993-1000.

12 Lala S, Ogura Y, Osborne C, et al. Crohn's disease and the NOD2 gene: a role for Paneth cells. Gastroenterology 2003; 125:47-57.

13 Lesage S, Zouali H, Cezard JP, et al. CARD15/ NOD2 mutational analysis and genotypephenotype correlation in 612 patients with inflammatory bowel disease. Am J Hum Genet 2002;70:845-57.

14 Ahmad T, Armuzzi A, Bunce M, et al. The molecular classification of the clinical manifestations of Crohn's disease. Gastroenterology 2002; 122:854-66.

15 Cuthbert AP, Fisher SA, Mirza MM, et al. The contribution of NOD2 gene mutations to the risk and site of disease in inflammatory bowel disease. Gastroenterology 2002;122:867-74.

16 Wehkamp J, Harder J, Weichenthal M, et al. NOD2 (CARD15) mutations in Crohn's disease are associated with diminished mucosal $\alpha$ defensin expression. Gut 2004;53:1658-64.

17 Yang D, Chen Q, Chertov O, et al. Human neutrophil defensins selectively chemoattract naive T and immature dendritic cells. J Leukoc Biol 2000;68:9-14.

18 Lillard JW jr, Boyaka PN, Chertov O, et al Mechanisms for induction of acquired host immunity by neutrophil peptide defensins. Proc Natl Acad Sci U S A 1999:96:651-6.

19 Tani K, Murphy WJ, Chertov O, et al. Defensins act as potent adjuvants that promote cellular and humoral immune responses in mice to a lymphoma idiotype and carrier antigens. Int Immunol 2000;12:691-700.

20 Ayabe T, Satchell DP, Wilson CL, et al. Secretion of microbicidal a-defensins by intestinal Paneth cells in response to bacteria. Nat Immunol 2000;1:113-18.

21 Garabedian EM, Roberts LJ, McNevin MS, et al. Examining the role of Paneth cells in the small intestine by lineage ablation in transgenic mice. J Biol Chem 1997;272:23729-40.

22 Salzman NH, Ghosh D, Huttner KM, et al. Protection against enteric salmonellosis in transgenic mice expressing a human intestinal defensin. Nature 2003;422:522-6. 